


\title{
Some Interfaces Between Noncommutative Ring Theory and Operator Algebras
}

\author{
Pere ARA
}

\begin{abstract}
In this talk, I will present a unified approach to several constructions in operator algebras that are related to the algebraic theory of rings of quotients, thus showing deep relations between algebraic and analytic concepts. These constructions include the algebra of unbounded operators affiliated to a finite von Neumann algebra, defined by Murray and von Neumann in 1936, and the $C^{*}$-algebra of essential multipliers of a $C^{*}$-algebra, defined by Elliott in 1976. In both cases, the algebras were defined without any explicit reference to localisation theory, but the fact that they can be obtained as algebras of quotients has played a very important role in several applications.
\end{abstract}

\section{INTRODUCTION}

A pre- $C^{*}$-algebra is a complex *-algebra $A$ endowed with a norm $\|\cdot\|$ satisfying $\|x y\| \leq\|x\|\|y\|$ for all $x, y \in A$ and the important $C^{*}$-equation

$$
\left\|x^{*} x\right\|=\|x\|^{2}
$$

for all $x \in A$. We call a norm satisfying the above properties a $C^{*}$ norm. A $C^{*}$-algebra is a pre- $C^{*}$-algebra which is complete for the uniform structure induced by the norm. Every pre- $C^{*}$-algebra $A$ can be completed, and so the pre- $C^{*}$-algebras are just the ${ }^{*}$-subalgebras of $C^{*}$-algebras. An important example of a $C^{*}$-algebra is the algebra $B(H)$ of all bounded linear operators on a Hilbert space $H$. The

Partially supported by grants from the DGICYT (Spain) and the Comissionat per Universitats i Recerca de la Generalitat de Catalunya. 
involution on this algebra is given by the adjoint operation and the norm is the operator norm:

$$
\|T\|=\sup _{\|x\| \leq 1}\|T x\| .
$$

A von Neumann algebra is a ${ }^{*}$-subalgebra $M$ of $B(H)$ such that $M=M^{\prime \prime}$, where, for a subset $X$ of $B(H)$, we denote by $X^{\prime}$ the commutant of $X$, that is, the set of elements of $B(H)$ that commute with every element in $X$. Every von Neumann algebra is a $C^{*}$ algebra, but not every $C^{*}$-algebra admits an ${ }^{*}$-isomorphism with a von Neumann algebra.

Many important $C^{*}$-algebras appear as completions of *-algebras under suitable $C^{*}$-norms on them. For example, both the group $C^{*}$ algebra $C^{*}(G)$ and the reduced group $C^{*}$-algebra $C_{r}^{*}(G)$ appear as the completion of $\mathbb{C}[G]$ once we put on it suitable $C^{*}$-norms. See Section 3 for the definition of the reduced group $C^{*}$-algebra.

The reader is referred to the textbooks $[\mathbf{1 6}],[\mathbf{3 0}],[\mathbf{3 5}]$ and $[\mathbf{3 6}]$ for more information on $C^{*}$-algebras.

Let $B$ be a complex unital *-algebra. The involution * is said to be positive-definite if, for every finite family $\left\{x_{i}\right\}$ of elements in $B$, the relation $\sum x_{i}^{*} x_{i}=0$ implies that $x_{i}=0$ for all $i$. The involution of a pre- $C^{*}$-algebra is always positive definite. We sketch here the general rule that we will follow to attach a $C^{*}$-algebra of quotients to any symmetric algebra of quotients of a $C^{*}$-algebra $A$. (In fact it is not necessary to start with a $C^{*}$-algebra to perform this construction, and some examples will be constructed in Section 3 which does not come from a $C^{*}$-algebra, but for the sake of simplicity let us restrict here to the case where $A$ is a $C^{*}$-algebra.) Assume that $Q(A)$ is a symmetric algebra of quotients of $A$ in the sense that the involution of $A$ can be extended to $Q(A)$. This happens with the classical ring of quotients of $A$ in case $A$ satisfies the left Ore condition (equivalently, the right Ore condition), and always with the symmetric Martindale algebra of quotients and the symmetric maximal algebra of quotients; see Section 2. Then the involution on $Q(A)$ will be positive definite and we can perform the Handelman-Vidav construction to $Q(A)$ to get the *-algebra of bounded elements $Q(A)_{b}$, as follows.

Let $Q=Q(A)$. Then $Q$ is a unital, complex *-algebra with positive-definite involution. We define the positive cone $Q_{+}$of $Q$ 
as the set of all elements of the form $\sum x_{i}^{*} x_{i}$, where $\left\{x_{i}\right\}$ is a finite subset of $Q$. The self-adjoint part $Q_{s a}$ of $Q$ becomes naturally a partially ordered real vector space by $x \leq y$ if $y-x \in Q_{+}$. An element $x \in Q$ is said to be bounded if $x^{*} x \leq n 1$ for some positive integer $n$. This is tantamount to the existence of a finite subset $\left\{y_{i}\right\}$ of $Q$ such that

$$
x^{*} x+\sum y_{i}^{*} y_{i}=n 1 .
$$

We will denote the set of all bounded elements of $Q$ by $Q_{b}$. It is not hard to see that $Q_{b}$ is a ${ }^{*}$-subalgebra of $Q$ in general, cf. [20] and [39]. There is a seminorm on $Q(A)_{b}$, which can be seen to be a norm in our situation, given by

$$
\|x\|^{2}=\inf \left\{\lambda \in \mathbb{R} \mid x^{*} x \leq \lambda 1\right\} .
$$

It is proved in $[\mathbf{2 0}]$ that $Q(A)_{b}$ is a pre- $C^{*}$-algebra with this norm. Its completion is therefore a $C^{*}$-algebra, and we call it a $C^{*}$-algebra of quotients of $A$. We hope to convince the reader that it is the analytic-algebraic context of the theory what makes it useful.

\section{The Commutative Case}

There is nothing more familiar to an algebraist than taking the field of fractions of a commutative domain. In case the commutative ring $R$ has zero-divisors, we invert the set of non zero-divisors and we obtain the so-called classical ring of quotients $Q_{c l}(R)$. If $A$ is a commutative von Neumann algebra then $A=L^{\infty}(X, \mu)$ for some locally compact Hausdorff space $X$ and some finite measure $\mu$ on $X$. Here $L^{\infty}(X, \mu)$ denotes the algebra of essentially bounded measurable functions modulo the ideal of measurable functions which vanish outside a subset of measure zero, with the norm given by the essential supremum. One can see that this is a maximal abelian subalgebra of the algebra $B\left(L^{2}(X, \mu)\right)$ of bounded linear operators on the Hilbert space $L^{2}(X, \mu)$. In this case we have $Q_{c l}(A)=L(X, \mu)$, the algebra of equivalence classes of measurable functions on $X$, and $Q_{c l}(A)_{b}=L^{\infty}(X, \mu)$, so that the $C^{*}$-algebra of quotients of $A$ is $A$ itself. The maximal ring of quotients of $A$ is also $L(X, \mu)$.

Now consider a general commutative $C^{*}$-algebra $A$. Then there exists a locally compact Hausdorff space $X$ such that $A=C_{0}(X)$, the 
algebra of continuous functions on $X$ which vanish at infinity, with the supremum norm. An element $f \in C_{0}(X)$ is not a zero-divisor if and only if the cozero set of $f$ is dense in $X$. Here $Q_{c l}(A)_{b} \neq A$ in general, for an easy example take the interval $X=[-1,1]$. Then the function $x /|x|$ is bounded but not continuous. If $X$ is metrisable, then $Q_{c l}(A)=\operatorname{alg} \lim _{U \in \mathcal{D}} C(U)$ and $Q_{c l}(A)_{b}=\operatorname{alg} \lim _{U \in \mathcal{D}} C_{b}(U)$, where $\mathcal{D}$ is the set $\overrightarrow{\text { of }}$ dense open subsets of $X$. In the non-metrisable case, the algebra alg $\lim _{U \in \mathcal{D}} C(U)$ can be bigger than $Q_{c l}(A)$, since $Q_{c l}(A)$ only involves the dense cozero sets in $X$. It turns out that alg $\lim _{U \in \mathcal{D}} C(U)$ is the so-called maximal ring of quotients of $C_{0}(X)$, denoted $Q_{\max }(A)$, and of course $\operatorname{alg} \lim _{U \in \mathcal{D}} C_{b}(U)$ is its bounded part. Since $Q_{\max }(A)$ is the quotient algebra that can be generalised to the non-commutative case, we will focus on it for the rest of this section. The completion of $Q_{\max }(A)_{b}$ is the $C^{*}$-direct limit $Q(A)=\lim _{U \in \mathcal{D}} C_{b}(U)$. It can also be described as the algebra of bounded Borel functions on $X$ modulo the ideal of bounded Borel functions that vanish outside of a set of the first category; see [3, Proposition 3.4.5]. The $C^{*}$-algebra $Q(A)$ should be thought of as a topological completion of $C(X)$ whilst the von Neumann algebra $L^{\infty}(X, \mu)$ is a measure-theoretic completion of $C(X)$. It is interesting to observe that $Q(A)$ has several important characterizations: It is the injective envelope of $A$ (see Section 2 for the definition), and it is also the regular monotone completion of $A$; see [14, Theorem 4.5] and [18, Theorem 6.3].

\section{The Local Multiplier Algebra}

The construction in Section 1 can be generalised in at least two different ways to the non-commutative situation. We first sketch the construction of the local multiplier algebra, based on the symmetric Martindale algebra of quotients, and then we consider a newer concept, based on the symmetric maximal algebra of quotients.

We need the notion of the multiplier algebra $M(A)$ of a semiprime algebra $A$. Roughly speaking this is the maximal unitisation of $A$. More precisely $M(A)$ is a unital algebra containing $A$ as an essential ideal, and for any unital algebra $T$ containing $A$ as an ideal, there is a unique unital algebra homomorphism $T \rightarrow M(A)$ which is the identity on $A$. (Recall that a (right) ideal $I$ of an algebra $T$ is said 
to be essential if for every non-zero (right) ideal $J$ of $A$ we have $I \cap J \neq 0$.) If $A=C_{0}(X)$ for some locally compact Hausdorff space

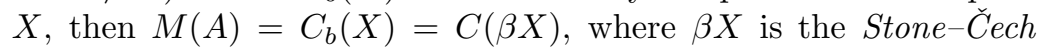
compactification of $X$. Now, the closed ideals of $C_{0}(X)$ are of the form $C_{0}(U)$, where $U$ is an open subset of $X$, and the closed essential ideals correspond to open and dense subsets, so that we have

$$
Q\left(C_{0}(X)\right)=\underline{\lim }_{U \in \mathcal{D}} C_{b}(U)=\underline{\lim }_{U \in \mathcal{D}} M\left(C_{0}(U)\right)=\underline{\lim }_{I \in \mathcal{I}_{c e}} M(I),
$$

where $\mathcal{I}_{c e}$ denotes the family of closed essential ideals of $A$. This makes it possible to define a $C^{*}$-algebra of quotients for a general non-commutative $C^{*}$-algebra $A$, called the local multiplier algebra of $A$, namely

$$
M_{\mathrm{loc}}(A)=\underline{\lim }_{I \in \mathcal{I}_{c e}} M(I) .
$$

In [3], Martin Mathieu and the author describe at large the many nice properties and applications of this algebra. It turns out that $M_{\text {loc }}(A)$ also follows the pattern we have sketched above for the classical and the maximal ring of quotients of a commutative $C^{*}$ algebra $A$. Note that in the non-commutative case the classical ring of quotients could very well not exist (the algebra can fail to satisfy Ore's condition), and the maximal ring of quotients is very large and it has the additional shortcoming that the involution does not extend to it. The local multiplier algebra corresponds rather to the symmetric Martindale ring of quotients of $A$. For the construction of this ring of quotients, see [21] (and also [32], [33] and [4] for the prime case). For the relationship with the local multiplier algebra, see $[\mathbf{3}$, Chapter 2].

The $C^{*}$-algebra $M_{\mathrm{loc}}(A)$ was first defined (using the direct limit formula above) by Elliott in 1976 [11], under the name of essential multipliers, and was used by him to study automorphisms and derivations on $A F$-algebras. Somewhat later, Pedersen proved in $[\mathbf{3 4}]$ that every derivation on a separable $C^{*}$-algebra becomes inner in $M_{\mathrm{loc}}(A)$.

The connection with the theory of rings of quotients was realised by Martin Mathieu and the speaker. They also realised, in analogy with algebra, the importance of the centre of $M_{\mathrm{loc}}(A)$. 
By a local version of the Dauns-Hofmann Theorem ([1] or $[\mathbf{3}])$, the centre $Z$ of $M_{\text {loc }}(A)$ is described as

$$
Z=\underline{\lim }_{U \in \mathcal{D}} C_{b}(U),
$$

where now $\mathcal{D}$ is the set of dense open subsets of the primitive spectrum of $A$.

The $C^{*}$-subalgebra $\overline{A Z}$ of $M_{\mathrm{loc}}(A)$ is called the bounded central closure of $A$ and it is denoted by ${ }^{c} A$. It plays an important role in the study of several problems related with operators on $C^{*}$-algebras, see [3]. As its algebraic analogue, ${ }^{c} A$ is a closure operation, i.e., ${ }^{\mathrm{c}}\left({ }^{c} A\right)={ }^{c} A$. The following result is a sample of the results obtained by using these techniques. It generalises a well-known result for von Neumann algebras.

Theorem 2.1. (Mathieu [27], [28]) For an inner derivation $\delta_{a}$ on a $C^{*}$-algebra $A$, we have

$$
\left\|\delta_{a}\right\|=2 \operatorname{dist}(a, Z)
$$

Moreover there is $b \in M_{\mathrm{loc}}(A)$ such that $\delta_{a}=\delta_{b}$ and $\left\|\delta_{b}\right\|=2\|b\|$.

Some important structural questions on $M_{\mathrm{loc}}(A)$ remain open:

(1) Is the construction of $M_{\mathrm{loc}}(A)$ a closure operation? (i.e., does the equality $M_{\mathrm{loc}}\left(M_{\mathrm{loc}}(A)\right)=M_{\mathrm{loc}}(A)$ hold?)

(2) Is every derivation on $M_{\mathrm{loc}}(A)$ inner? (Not known even for separable $A$.)

(3) Characterise the $C^{*}$-algebras $A$ such that $M_{\text {loc }}(A)$ is simple.

A positive solution to (2) would give a unified approach to two results by Sakai, one stating that every derivation on a simple $C^{*}$ algebra is inner in its multiplier algebra, and the other saying that every derivation on a von Neumann algebra is inner. Some advances on Problems (1) and (2) have been obtained by Somerset in [37]. For example, it is shown that if $A$ is a unital separable $C^{*}$-algebra such that $\operatorname{Prim}(A)$ has a dense $G_{\delta}$-subset consisting of closed points, then $M_{\text {loc }}(A)$ is its own local multiplier algebra and has only inner derivations [37, Theorem 2.7]. Problem (3) has been raised by [2], where the first non-trivial examples of $C^{*}$-algebras with simple local 
multiplier algebra were constructed. We believe that the structure of the lattice of ideals of $M_{\mathrm{loc}}(A)$ deserves further investigation.

Various aspects of the theory of local multipliers have been surveyed in the papers $[\mathbf{2 5}, \mathbf{2 6}, \mathbf{2 8}, \mathbf{2 9}]$.

Now we consider the symmetric maximal algebra of quotients of a $C^{*}$-algebra $A$. The corresponding algebraic ring of quotients has been considered in depth only recently in $[\mathbf{2 2}]$. The construction is based on essential right ideals $I$ of $A$. Note that for an essential right ideal $I$ of $A$ we have $\ell_{A}(I)=0$, where $\ell_{A}(X)$ is the left annihilator of a subset $X$ of $A$. Indeed, if $x$ is a non-zero element in $A$, then there is $z \in A$ such that $x^{*} z$ is a non-zero element in $I$ by the essentiality of $I$, and so $\left(x^{*} z\right)^{*}\left(x^{*} z\right) \neq 0$, showing that $x I \neq 0$. Let $\mathcal{I}_{\text {er }}$ denote the family of essential right ideals of $A$.

Consider triples $(f, g, I)$, where $I \in \mathcal{I}_{\text {er }}, f: I^{*} \rightarrow A$ is a left $A$ module homomorphism, $g: I \rightarrow A$ is a right $A$-module homomorphism and they satisfy the compatibility rule

$$
f(x) y=x g(y)
$$

for all $x \in I^{*}$ and $y \in I$. Two such triples $\left(f_{1}, g_{1}, I_{1}\right)$ and $\left(f_{2}, g_{2}, I_{2}\right)$ are equivalent if $g_{1}$ and $g_{2}$ coincide on $I_{1} \cap I_{2}$. In that case we have, for all $x, y \in I_{1} \cap I_{2}$,

$$
0=x^{*}\left(g_{1}(y)-g_{2}(y)\right)=\left(f_{1}\left(x^{*}\right)-f_{2}\left(x^{*}\right)\right) y,
$$

so that $f_{1}$ and $f_{2}$ agree on $I_{1}^{*} \cap I_{2}^{*}$, because $I_{1} \cap I_{2}$ is an essential right ideal. This also shows that the first component in the triple $(f, g, I)$ is determined by the second, so what is really crucial is that we impose the existence of $f$. Let $Q_{\sigma}(A)$ be the set of all equivalence classes of triples $(f, g, I)$. We define algebraic operations on $Q_{\sigma}(A)$ by

$$
\begin{aligned}
{\left[\left(f_{1}, g_{1}, I_{1}\right)\right]+\left[\left(f_{2}, g_{2}, I_{2}\right)\right] } & =\left[\left(h_{1}, h_{2}, I_{1} \cap I_{2}\right)\right], \\
{\left[\left(f_{1}, g_{1}, I_{1}\right)\right]\left[\left(f_{2}, g_{2}, I_{2}\right)\right] } & =\left[\left(k_{1}, k_{2}, J\right)\right], \\
{[(f, g, I)]^{*} } & =\left[\left(g^{*}, f^{*}, I\right)\right] .
\end{aligned}
$$

Here, $h_{1}(x)=f_{1}(x)+f_{2}(x)$ and $h_{2}(y)=g_{1}(y)+g_{2}(y)$, while $J=$ $\left(f_{1}^{-1}\left(I_{2}^{*}\right)^{*}\right) \cap\left(g_{2}^{-1}\left(I_{1}\right)\right)$ and $k_{1}(x)=f_{2}\left(f_{1}(x)\right), k_{2}(y)=g_{1}\left(g_{2}(y)\right)$. 
Finally $f^{*}(x)=f\left(x^{*}\right)^{*}$ for all $x \in I$ and $g^{*}(x)=g\left(x^{*}\right)^{*}$ for all $x \in$ $I^{*}$. Endowed with these operations, $Q_{\sigma}(A)$ becomes a complex *algebra called the maximal symmetric algebra of quotients of $A[\mathbf{2 2}]$. We have an injective ${ }^{*}$-ring homomorphism $\iota: A \rightarrow Q_{\sigma}(A)$ given by $\iota(a)=\left(R_{a}, L_{a}, A\right)$, where $R_{a}$ (resp. $\left.L_{a}\right)$ denotes right (resp. left) multiplication by $a$. Since the symmetric maximal ring of quotients of a $C^{*}$-algebra coincides with that of its unitisation, we will assume in the rest of this section that $A$ is a unital $C^{*}$-algebra.

We will not apply the general Handelman-Vidav process to $Q_{\sigma}(A)$, but a closely related one, which coincides with it in many situationsin particular in the commutative case. Our $C^{*}$-algebra of quotients associated with $Q_{\sigma}(A)$ will be related to the hereditary $C^{*}$ subalgebras of $A$. Recall that there is a bijective, order-preserving correspondence between the set of closed right ideals of $A$ and the set of hereditary $C^{*}$-subalgebras of $A$, given by assigning to a closed right ideal $I$ the hereditary $C^{*}$-subalgebra $I \cap I^{*}$ and to a hereditary $C^{*}$-subalgebra $D$ the right ideal $I=\left\{x \in A \mid x x^{*} \in D\right\}$; see [35, Theorem 1.5.2]. For a subset $S$ in $A$ define

$$
S^{\perp}=\{x \in A \mid S x=x S=0\},
$$

the annihilator of $S$. We say that a hereditary $C^{*}$-subalgebra $D$ of $A$ is essential in case the right ideal generated by $D$ is essential as a right ideal. The above bijective correspondence restricts to a bijection between the set of closed right ideals $I$ of $A$ such that $\ell_{A}(I)=0$ and the set of hereditary $C^{*}$-subalgebras $D$ of $A$ such that $D^{\perp}=0$. This further restricts to a bijective correspondence between the set $\mathcal{I}_{c e r}$ of closed essential right ideals and the set $\mathcal{H}_{e}$ of essential hereditary $C^{*}$-subalgebras of $A$.

We start by defining a positive cone $P$ in $Q_{\sigma}(A)$. An element $\alpha$ in $Q_{\sigma}(A)$ belongs to $P$, by definition, in the case $\alpha$ has a representative $(f, g, I)$ such that

$$
f\left(x^{*}\right) x=x^{*} g(x) \geq 0
$$

for all $x \in I$. The first important observation is the following.

Lemma 2.2. For an element $a \in A$ we have $a \geq 0$ in $A$ if and only if $\iota(a) \in P$.

Proof. Clearly $\iota(a) \in P$ if $a \geq 0$ in $A$. Assume that $\iota(a) \in P$. Then there exists an essential right ideal $I$ of $A$ such that $x^{*} a x \geq 0$ for 
all $x \in I$. Without loss of generality we can assume that $I$ is closed. Assume first that $a=a^{*}$. Then we can write $a=a_{+}-a_{-}$, with $a_{+}$ and $a_{-}$positive and $a_{+} a_{-}=0$. Multiplying the above relation by $a_{-}^{1 / 2}$ on the right and on the left, we get $a_{-}^{1 / 2} a a_{-}^{1 / 2}=-a_{-}^{2} \leq 0$. On the other hand $J:=\left(a_{-}^{1 / 2}\right)^{-1} I=\left\{x \in A \mid a_{-}^{1 / 2} x \in I\right\}$ is an essential closed ideal of $A$, and for $x$ in $J$ we have

$$
0 \leq\left(a_{-}^{1 / 2} x\right)^{*} a\left(a_{-}^{1 / 2} x\right)=-x^{*} a_{-}^{2} x \leq 0 .
$$

From this we conclude that $x^{*} a_{-}^{2} x=0$ and so $a_{-}=0$ because $J$ is an essential right ideal. An analogous argument shows that, if $b=b_{+}-b_{-}$is self-adjoint and $x^{*} b x=0$ for all $x \in I$ then $b_{+}=b_{-}=0$ and so $b=0$. Now assume $a \in A$ is a general element such that $x^{*} a x \geq 0$ for all $x \in I$. Then $x^{*}\left(\frac{a-a^{*}}{2 i}\right) x=0$ for all $x \in I$ and so $a-a^{*}=0$ by the above observation. Therefore $a=a^{*}$ and the first part of the argument gives us $a \geq 0$, as desired.

An element $\alpha$ in $Q_{\sigma}(A)$ is said to be bounded in case there exists a positive real number $\lambda$ such that $\lambda^{2} \cdot 1-\alpha^{*} \alpha \in P$ and $\lambda^{2} \cdot 1-\alpha \alpha^{*} \in P$. We denote by $Q_{\sigma}(A)_{b}$ the set of bounded elements in $Q_{\sigma}(A)$. For $\alpha$ in $Q_{\sigma}(A)_{b}$ define

$$
\|\alpha\|_{\sigma}=\inf \left\{\lambda \in \mathbb{R}_{+} \mid \lambda^{2} \cdot 1-\alpha^{*} \alpha \in P \text { and } \lambda^{2} \cdot 1-\alpha \alpha^{*} \in P\right\} .
$$

The following properties are easily checked. (For (P4) one needs Lemma 2.2.)

(P1) If $\alpha, \beta \in P$ then $\alpha+\beta \in P$.

(P2) If $\alpha \in P$ and $v \in \mathbb{R}_{+}$then $v \alpha \in P$.

(P3) If $\alpha \in P$ and $\beta \in Q_{\sigma}(A)$ then $\beta^{*} \alpha \beta \in P$.

(P4) For $a \in A$ we have $\|a\|=\|\iota(a)\|_{\sigma}$.

Properties $(\mathrm{P} 1)-(\mathrm{P} 2)$ say that $P$ is a cone in $Q_{\sigma}(A)$. Now we observe that if $\alpha \in Q_{\sigma}(A)_{b}$ then for every representative $(f, g, I)$ of $\alpha$, the maps $f$ and $g$ are bounded linear maps with $\|f\|,\|g\| \leq$ $\|\alpha\|_{\sigma}$. Indeed, given such a representative $(f, g, I)$ of $\alpha$ and given $\lambda>\|\alpha\|_{\sigma}$, there is an essential right ideal $J$ such that $J \subseteq I$ and $x^{*} f^{*}(g(x)) \leq \lambda^{2} x^{*} x$ and $x^{*} g\left(f^{*}(x)\right) \leq \lambda^{2} x^{*} x$ for all $x \in J$. But $x^{*} f^{*}(g(x))=g^{*}\left(x^{*}\right) g(x)=g(x)^{*} g(x)$ and so we get $g(x)^{*} g(x) \leq$ $\lambda^{2} x^{*} x$. By $[\mathbf{3 5}, 1.3 .5]$, we obtain $\|g(x)\| \leq \lambda\|x\|$ and so $g$ is bounded on $J$ with $\left\|g_{\mid J}\right\| \leq \lambda$. Similarly we obtain that $f$ is bounded on $J^{*}$ 
with $\left\|f_{\mid J^{*}}\right\| \leq \lambda$. It follows easily from Lemma 2.2 that for $a \in A$ we have

$$
\|a\|=\left\|L_{a \mid J}\right\|=\left\|R_{a \mid J^{*}}\right\| .
$$

On the other hand, for $x \in I$ and $y \in J$ with $\|y\| \leq 1$, we have

$$
\left\|y^{*} g(x)\right\|=\left\|f\left(y^{*}\right) x\right\| \leq\left\|f_{\mid J^{*}}\right\|\|x\| \leq \lambda\|x\| .
$$

It follows from the above that $\|g(x)\| \leq \lambda\|x\|$ for all $x \in I$ and so $\|g\| \leq \lambda$. Since this holds for all $\lambda>\|\alpha\|_{\sigma}$, we get $\|g\| \leq\|\alpha\|_{\sigma}$, and similarly $\|f\| \leq\|\alpha\|_{\sigma}$.

Therefore every element $\alpha$ in $Q_{\sigma}(A)_{b}$ has a representative where the functions are defined on closed essential right ideals. Let $\mathcal{I}_{c e r}$ denote the set of closed essential right ideals of $A$. As mentioned before, this set is in bijective correspondence with the set $\mathcal{H}_{e}$ of essential hereditary $C^{*}$-subalgebras of $A$.

Theorem 2.3. For every $C^{*}$-algebra $A, Q_{\sigma}(A)_{b}$ is a ${ }^{*}$-algebra and $\|\cdot\|_{\sigma}$ is a $C^{*}$-norm on it.

Proof. We have to prove the following properties:

(1) For $\alpha, \beta \in Q_{\sigma}(A)_{b}$, we have $\|\alpha+\beta\|_{\sigma} \leq\|\alpha\|_{\sigma}+\|\beta\|_{\sigma}$. In particular $\alpha+\beta \in Q_{\sigma}(A)_{b}$.

(2) For $\alpha \in Q_{\sigma}(A)_{b}$ and $v \in \mathbb{C}$ we have $\|v \alpha\|_{\sigma}=|v|\|\alpha\|_{\sigma}$.

(3) For $\alpha, \beta \in Q_{\sigma}(A)_{b}$ we have $\|\alpha \beta\|_{\sigma} \leq\|\alpha\|_{\sigma}\|\beta\|_{\sigma}$. In particular $\alpha \beta \in Q_{\sigma}(A)_{b}$.

(4) For $\alpha \in Q_{\sigma}(A)_{b}$ we have $\left\|\alpha^{*}\right\|_{\sigma}=\|\alpha\|_{\sigma}$. In particular, $\alpha^{*} \in$ $Q_{\sigma}(A)_{b}$.

(5) For $\alpha \in Q_{\sigma}(A)_{b}$ we have $\left\|\alpha^{*} \alpha\right\|_{\sigma}=\|\alpha\|_{\sigma}^{2}$.

(6) For $\alpha \in Q_{\sigma}(A)_{b}$ we have $\|\alpha\|_{\sigma}=0$ if and only if $\alpha=0$.

By our previous observations, we can take representatives $(f, g, I)$ for elements in $Q_{\sigma}(A)_{b}$ with $I$ a closed essential right ideal of $A$.

Proof of (1): Put $\alpha=\left[\left(f_{1}, g_{1}, I_{1}\right)\right]$ and $\beta=\left[\left(f_{2}, g_{2}, I_{2}\right)\right]$, with $I_{1}, I_{2} \in \mathcal{I}_{\text {cer }}$. Take $\lambda>\|\alpha\|_{\sigma}$ and $\mu>\|\beta\|_{\sigma}$. Set $J=I_{1} \cap I_{2}$. For all $x \in J$ we have

$$
\begin{aligned}
x^{*}\left[(\lambda+\mu)^{2} 1-\right. & \left.\left(f_{1}+f_{2}\right)^{*}\left(g_{1}+g_{2}\right)\right](x) \\
= & \left(\lambda^{2} x^{*} x-x^{*} f_{1}^{*}\left(g_{1}(x)\right)\right)+\left(\mu^{2} x^{*} x-x^{*} f_{2}^{*}\left(g_{2}(x)\right)\right) \\
& +2 \lambda \mu x^{*} x-x^{*} f_{1}^{*}\left(g_{2}(x)\right)-x^{*} f_{2}^{*}\left(g_{1}(x)\right) \\
\geq & 2 \lambda \mu x^{*} x-x^{*} f_{1}^{*}\left(g_{2}(x)\right)-x^{*} f_{2}^{*}\left(g_{1}(x)\right) .
\end{aligned}
$$


Therefore we have to prove that $g_{1}(x)^{*} g_{2}(x)+g_{2}(x)^{*} g_{1}(x) \leq 2 \lambda \mu x^{*} x$ for all $x \in J$. Put $D=J \cap J^{*}$ and take an approximate identity $\left(u_{i}\right)$ for $D$. For $x \in J$ and each $i$ we have

$$
\begin{aligned}
g_{1}\left(u_{i} x\right)^{*} g_{2}\left(u_{i} x\right) & +g_{2}\left(u_{i} x\right)^{*} g_{1}\left(u_{i} x\right) \\
& =x^{*}\left[g_{1}\left(u_{i}\right)^{*} g_{2}\left(u_{i}\right)+g_{2}\left(u_{i}\right)^{*} g_{1}\left(u_{i}\right)\right] x \\
& \leq x^{*}(2 \lambda \mu) x=2 \lambda \mu x^{*} x .
\end{aligned}
$$

Since $g_{1}$ and $g_{2}$ are continuous and $x=\lim _{i} u_{i} x$ for $x \in J$, we get

$$
\begin{aligned}
g_{1}(x)^{*} g_{2}(x)+g_{2}(x)^{*} g_{1}(x) & =\lim _{i}\left[g_{1}\left(u_{i} x\right)^{*} g_{2}\left(u_{i} x\right)+g_{2}\left(u_{i} x\right)^{*} g_{1}\left(u_{i} x\right)\right] \\
& \leq 2 \lambda \mu x^{*} x
\end{aligned}
$$

as desired.

(2) is easy and left to the reader.

Proof of (3): Take $\lambda>\|\alpha\|_{\sigma}$ and $\mu>\|\beta\|_{\sigma}$ and take suitable representatives $\left(f_{1}, g_{1}, I_{1}\right)$ and $\left(f_{2}, g_{2}, I_{2}\right)$ of $\alpha$ and $\beta$, respectively. Set $J:=\left(g_{2}^{-1}\left(I_{1}\right)\right) \cap\left(f_{1}^{-1}\left(I_{2}^{*}\right)^{*}\right)$. For $x \in J$ we have

$$
g_{1}\left(g_{2}(x)\right)^{*} g_{1}\left(g_{2}(x)\right) \leq \lambda^{2} g_{2}(x)^{*} g_{2}(x) \leq \lambda^{2} \mu^{2} x^{*} x .
$$

We conclude that $\lambda^{2} \mu^{2}-(\alpha \beta)^{*}(\alpha \beta) \in P$. Similarly $\lambda^{2} \mu^{2}-(\alpha \beta)(\alpha \beta)^{*}$ belongs to $P$ so that $\|\alpha \beta\|_{\sigma} \leq\|\alpha\|_{\sigma}\|\beta\|_{\sigma}$, as wanted.

Property (4) is obvious from the definitions. Taking into account properties (3) and (4), in order to prove (5) it is enough to show $\|\alpha\|_{\sigma}^{2} \leq\left\|\alpha^{*} \alpha\right\|_{\sigma}$. The latter inequality is proved exactly as in [39, page 78].

Proof of (6): If $\alpha=[(f, g, I)] \in Q_{\sigma}(A)_{b}$ then $\alpha \iota(x)=\iota(g(x))$ for every $x \in I$. Since $\|a\|=\|\iota(a)\|_{\sigma}$ for $a \in A$ we get

$$
\|g(x)\|=\|\alpha \iota(x)\|_{\sigma} \leq\|\alpha\|_{\sigma}\|x\|
$$

and so $\|\alpha\|_{\sigma}=0$ implies that $g=0$ and $\alpha=0$.

Let $Q_{h}(A)$ be the $C^{*}$-completion of $Q_{\sigma}(A)$ under the norm $\|\cdot\|_{\sigma}$.

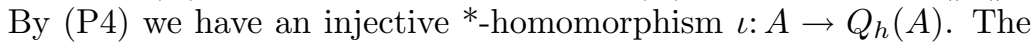
following lemma is quite useful. 
Lemma 2.4. Let $A$ be a $C^{*}$-algebra, and let $\alpha=[(f, g, I)] \in Q_{\sigma}(A)_{b}$, with $I$ a closed essential right ideal of $A$. Let $\left(u_{i}\right)$ be an approximate identity for $D=I \cap I^{*}$. Then

$$
\|\alpha\|_{\sigma}=\|f\|=\|g\|=\sup _{i}\left\|g\left(u_{i}\right)\right\|=\sup _{i}\left\|f\left(u_{i}\right)\right\| .
$$

Proof. Clearly $\sup _{i}\left\|f\left(u_{i}\right)\right\|=\|f\|$ and $\sup _{i}\left\|g\left(u_{i}\right)\right\|=\|g\|$. Since we have observed before that $\|f\|,\|g\| \leq\|\alpha\|_{\sigma}$, we only have to show that $\|\alpha\|_{\sigma} \leq \sup _{i}\left\|g\left(u_{i}\right)\right\|$. (The corresponding statement for $f$ follows by symmetry.) Take a positive real number $\lambda$ such that $\left\|g\left(u_{i}\right)\right\| \leq \lambda$ for all $i$. Then

$$
g\left(u_{i}\right)^{*} g\left(u_{i}\right) \leq \lambda^{2} \cdot 1
$$

and so

$$
g\left(u_{i} x\right)^{*} g\left(u_{i} x\right)=x^{*} g\left(u_{i}\right)^{*} g\left(u_{i}\right) x \leq \lambda^{2} x^{*} x
$$

for all $x \in I$ and all $i$, which proves that $\lambda^{2} x^{*} x-g(x)^{*} g(x) \geq 0$ and so $\lambda^{2} \cdot 1-\alpha^{*} \alpha \in P$. For all $x \in I^{*}$ with $\|x\| \leq 1$ and all $y \in I$ we have

$$
y^{*} f(x)^{*} f(x) y=g(y)^{*} x^{*} x g(y) \leq g(y)^{*} g(y) \leq \lambda^{2} y^{*} y .
$$

We infer that $\|f(x) y\| \leq \lambda\|y\|$ and so $\|f(x)\|=\left\|\left(L_{f(x)}\right)_{\mid I}\right\| \leq \lambda$ for all $x \in I^{*}$ with $\|x\| \leq 1$. Therefore $\|f\| \leq \lambda$ and so we conclude as before that $\lambda^{2} \cdot 1-\alpha \alpha^{*} \in P$. It follows that $\|\alpha\|_{\sigma} \leq \lambda$, and our proof is complete.

The above result tells us that we can build elements in $Q_{\sigma}(A)_{b}$ from suitable operators on essential hereditary $C^{*}$-subalgebras $D$ of $A$, with controlled norm. Namely assume that $f$ and $g$ are bounded linear operators from $D$ to $A$ such that $f(x) y=x g(y)$ for all $x, y \in$ $D$. Then we can uniquely extend $f$ (respectively, $g$ ) to a left (respectively, right) $A$-module homomorphism from the left (respectively, right) ideal generated by $D$, so that we get an element $\alpha$ in $Q_{\sigma}(A)_{b}$ with $\|\alpha\|_{\sigma}=\|f\|=\|g\|$. In particular there is a unital injective *homomorphism from the multiplier algebra $\mathcal{M}(D)$ to $Q_{h}(A)$. It also follows that we have a unital injective *-homomorphism $M_{\text {loc }}(A) \rightarrow$ 
$Q_{h}(A)$ which is the identity on $A$. (See below for a proof of this using the universal property of $Q_{h}(A)$.)

An enlargement of a $C^{*}$-algebra $A$ is a pair $(B, \kappa)$, where $B$ is a $C^{*}$-algebra and $\kappa: A \rightarrow B$ is a ${ }^{*}$-monomorphism. Let $A \subseteq B$ be an enlargement of $A$ (we suppress $\kappa$ for the sake of simplicity). For each $I \in \mathcal{I}_{\text {cer }}$ define an $A$-subbimodule $B_{I}^{A}$ of $B$ by

$$
B_{I}^{A}=\left\{b \in B \mid b I+b^{*} I \subseteq A\right\} .
$$

Set $B_{\mathcal{I}_{c e r}}=\bigcup_{I \in \mathcal{I}_{c e r}} B_{I}^{A}$. Then $B_{\mathcal{I}_{c e r}}$ is a ${ }^{*}$-subalgebra of $B$ and its completion is a $C^{*}$-algebra, which we denote by $B_{h}$. We say that $B$ is an $\mathcal{I}_{c e r}$-enlargement of $A$ in case $B=B_{h}$. An $\mathcal{I}_{c e r}$-enlargement $B$ of $A$ is said to be essential in case, for $q \in B$ and $I \in \mathcal{I}_{\text {cer }}$, we have $q I=0$ implies $q=0$. Note that the enlargement $\left(Q_{h}(A), \iota\right)$ is an essential $\mathcal{I}_{c e r}$-enlargement. Indeed, for $q \in Q_{h}(A)$ we can find a sequence $\left(q_{n}\right)$ in $Q_{\sigma}(A)_{b}$ converging to $q$. Without loss of generality we can assume that the domain of $q_{n}$ is contained in $I$ for all $n \geq 1$. From the fact that the norm of $q_{n}$ is determined by its action on its domain $I_{n}$ (Lemma 2.4) we conclude that $q I_{n} \neq 0$ for $n$ big enough. In particular $q I \neq 0$.

Let us now state the universal property of the algebra $Q_{h}(A)$ in a completely analogous fashion to the corresponding universal property of $M_{\mathrm{loc}}(A)$, see $[\mathbf{3}]$.

Proposition 2.5. (Universal property of $Q_{h}(A)$ ) Let $A \subseteq B$ be an enlargement of a $C^{*}$-algebra $A$ by a $C^{*}$-algebra $B$. Then there exists a unique *-homomorphism $\psi: B_{h} \rightarrow Q_{h}(A)$ which is the identity on $A$. The map $\psi$ is injective if and only if $B_{h}$ is an essential

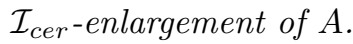

Proof. Define $\psi: B_{\mathcal{I}_{c e r}} \rightarrow Q_{\sigma}(A)_{b}$ by $\psi(b)=\left[\left(R_{b}, L_{b}, I\right)\right]$, where $b \in$ $B_{I}^{A}$. Clearly $\psi$ is a ${ }^{*}$-homomorphism, and we have to check that $\|\psi(b)\|_{\sigma} \leq\|b\|$ for all $b \in B_{\mathcal{I}_{\text {cer }}}$. Assume that $b \in B_{I}^{A}$ and set $\lambda=$ $\|b\|$. Then $\lambda^{2} \cdot 1-b^{*} b \geq 0$ in $B$, and so $\lambda^{2} x^{*} x-x^{*} b^{*} b x \geq 0$ for all $x \in I$, which gives $\lambda^{2} \cdot 1-\psi(b)^{*} \psi(b) \in P$. Similarly $\lambda^{2} \cdot 1-\psi(b) \psi(b)^{*} \in P$, and we conclude that $\|\psi(b)\|_{\sigma} \leq\|b\|$. We can therefore extend $\psi$ to a *-homomorphism (still denoted by $\psi$ ) from $B_{h}$ into $Q_{h}(A)$.

Note that Lemma 2.4 implies that the kernel of $\psi$ is precisely the set of elements $b \in B_{h}$ such that $b I=0$ for some closed essential 
right ideal $I$ of $A$. In particular, we see that $\psi$ is injective if and only if $B_{h}$ is an essential $\mathcal{I}_{c e r}$-enlargement of $A$.

Finally we indicate the connection of $Q_{h}(A)$ with the injective envelope $I(A)$ of a $C^{*}$-algebra, introduced by Hamana in [17]. Recall that $I(A)$ is an injective $C^{*}$-algebra, meaning that, given any self-adjoint linear subspace $S$ containing 1 of a $C^{*}$-algebra $C$, any completely positive linear map of $S$ into $I(A)$ extends to a completely positive linear map of $C$ into $I(A)$. Moreover the identity map is the only completely positive extension of the inclusion map $A \subseteq I(A)$. Every injective $C^{*}$-algebra is monotone complete and, in particular, an $A W^{*}$-algebra. We need the following lemma, which is proved in exactly the same way as the corresponding fact for essential ideals, see [14, Lemma 4.1].

Lemma 2.6. Let $D \in \mathcal{H}_{e}$. Then we have

$$
\{y \in I(A) \mid y D=0\}=\{y \in I(A) \mid D y=0\}=0 .
$$

Theorem 2.7. Let $A$ be a $C^{*}$-algebra. Then the $C^{*}$-algebra $Q_{h}(A)$ is ${ }^{*}$-isomorphic to $I(A)_{h}$. Moreover

$$
Z(I(A))=Z\left(Q_{h}(A)\right)=Z\left(M_{\mathrm{loc}}(A)\right) .
$$

Proof. By Proposition 2.5, there exists a unique *-homomorphism $\psi: I(A)_{h} \rightarrow Q_{h}(A)$ which is the identity on $A$. We first show that it is surjective. Let $I$ be a closed essential right ideal of $A$, and let $\alpha=[(f, g, I)]$ be in $Q_{\sigma}(A)_{b}$. Let $\left(u_{i}\right)$ be an approximate identity for $D=I \cap I^{*}$. Lemma 2.4 implies that

$$
\|f\|_{c b}=\|g\|_{c b}=\sup _{i}\left\|g\left(u_{i}\right)\right\|=\sup _{i}\left\|f\left(u_{i}\right)\right\|=\|\alpha\|_{\sigma} .
$$

By [14, Theorem 2.1], there is an element $y \in I(A)$ such that $g(x)=$ $y x$ for all $x \in I$ and $\|y\|=\|g\|_{c b}$. By Lemma 2.6, there is only one $y \in I(A)$ with this property. Now observe that

$$
(f(x)-x y) z=x(g(z)-y z)=0
$$

for all $x \in I^{*}$ and all $z \in I$. Consequently $f(x)=x y$ for all $x \in I^{*}$, and we conclude that $\psi(y)=\alpha$. This shows that $\psi$ is surjective, and $\psi$ is also injective because of Lemma 2.6 and Proposition 2.5. 
By $[\mathbf{1 9}], Z\left(M_{\text {loc }}(A)\right)=Z(I(A))$. We therefore conclude from $[\mathbf{1 7}]$ that $Z\left(M_{\mathrm{loc}}(A)\right)=Z\left(Q_{h}(A)\right)=Z(I(A))$.

We close with some questions on $Q_{h}(A)$ :

(1) We can prove that if $A$ is an $A W^{*}$-algebra then $A=Q_{h}(A)$. Is it true that $Q_{h}(A)$ is always an $A W^{*}$-algebra?

(2) Is it true that $Q_{h}\left(Q_{h}(A)\right)=Q_{h}(A)$ for every $C^{*}$-algebra $A$ ? (Conjecture: Yes.)

(3) Can we use $Q_{h}(A)$ to shed some light on the longstanding problem: Is every $A W^{*}$-algebra monotone complete?

\section{Group Algebras}

In this section we consider complex group algebras. A useful tool in the study of algebraic properties of these algebras comes from functional analysis, and we will describe some of the connections, in particular the work of Linnell on the zero-divisor conjecture (over the complex numbers). Recall that the zero-divisor conjecture states that a group algebra $K[G]$ has no non-trivial zero-divisors when $G$ is a torsion-free group. It turns out that localisation techniques in the analytic setting are fundamental.

For any discrete group $G$ we consider the Hilbert space $\ell^{2}(G)$ and embed the complex group algebra $\mathbb{C}[G]$ into the algebra $B\left(\ell^{2}(G)\right)$ via the left regular representation. The weak closure of $\mathbb{C}[G]$ in $B\left(\ell^{2}(G)\right)$ is the von Neumann group algebra $\mathcal{N}(G)$. It can be easily described as the algebra of right $G$-equivariant bounded linear operators on $\ell^{2}(G)$, that is, an element $T \in B\left(\ell^{2}(G)\right)$ belongs to $\mathcal{N}(G)$ if and only if

$$
T(\xi g)=T(\xi) g
$$

for every $\xi \in \ell^{2}(G)$ and $g \in G$. The group von Neumann algebra $\mathcal{N}(G)$ is a finite von Neumann algebra, meaning that it admits a faithful finite trace. In fact there is a canonical trace on $\mathcal{N}(G)$ given by the formula

$$
\operatorname{tr}(T)=\langle T e \mid e\rangle
$$

for every $T \in \mathcal{N}(G)$. For every finite von Neumann algebra $\mathcal{M}$, Murray and von Neumann constructed in [31] the algebra $\mathcal{U}$ of unbounded operators affiliated to $\mathcal{M}$, and they showed that this algebra is a von Neumann regular ring. It was observed by Berberian that 
$\mathcal{U}$ is both the classical ring of quotients and the maximal ring of quotients of $\mathcal{M}$; see [5] and [6]. The algebra of unbounded operators affiliated to the von Neumann group algebra $\mathcal{N}(G)$ is denoted by $\mathcal{U}(G)$.

Similarly to the commutative case (where $\mathcal{M}=L^{\infty}(X, \mu)$ and $\mathcal{U}=$ $L(X, \mu)$ ), we have $\mathcal{U}_{b}=\mathcal{M}$ for every finite von Neumann algebra $\mathcal{M}$.

We can now describe in some detail Linnell's result on the zerodivisor conjecture. We will denote by $\mathcal{E}$ the class of elementary amenable groups, that is, the minimal class of groups which contains all abelian groups and all finite groups and which is closed under extensions and direct limits. The Linnell class, denoted by $\mathcal{L}$, is the class of groups consisting on those groups $G$ which have a free normal subgroup $H$ such that the group $G / H$ is elementary amenable.

For an extension of rings $R \subset S$, the division closure of $R$ in $S$ is the smallest subring $T$ of $S$ containing $R$ and closed under inversion, that is, if $x \in T$ and $x$ is invertible in $S$, then $x^{-1} \in T$. The division closure of $\mathbb{C}[G]$ in $\mathcal{U}(G)$ will be denoted by $D(G)$.

Theorem 3.1. (Linnell [23]) Let $G$ be a torsion-free group in the Linnell class $\mathcal{L}$, and let $D$ be the division closure of $\mathbb{C}[G]$ in $\mathcal{U}(G)$. Then $D$ is a division ring. In particular $\mathbb{C}[G]$ is a domain.

The fact that $\mathcal{U}(G)$ is the classical ring of quotients of $\mathcal{N}(G)$ and a von Neumann regular ring is fundamental here. It is interesting to notice that, in the case where $G$ is a free group, the division ring $D$ one gets in Linnell's Theorem is the so-called free field ([7, p. 224]). This is in fact a crucial ingredient in Linnell's proof. The free field can be described in terms of localisation by means of what is known as universal localisation, a theory developed by P. M. Cohn. Namely given a ring $R$ and a set of square matrices $\Sigma$ with coefficients in $R$, one can form a ring $R_{\Sigma}$ obtained by universally inverting the matrices in $\Sigma$. More precisely, there exists a ring $R_{\Sigma}$ and a ring homomorphism $\varphi: R \rightarrow R_{\Sigma}$ such that (1) For every matrix $A \in \Sigma$ the matrix $\varphi(A)$ is invertible over $R_{\Sigma}$, and (2) Given a ring $T$ and a ring homomorphism $\psi: R \rightarrow T$ such that for every $A \in \Sigma$ the matrix $\psi(A)$ is invertible over $T$, there exists a unique ring homomorphism $\bar{\psi}: R_{\Sigma} \rightarrow T$ such that $\psi=\bar{\psi} \varphi$.

Now consider the group algebra of the free group $R=\mathbb{C}[H]$, and let $\Sigma$ be the set of all full square matrices over $R$. Here an square 
matrix $A \in M_{n \times n}(R)$ is called full if it cannot be written as a product $B C$, where $B \in M_{n \times(n-1)}(R)$ and $C \in M_{(n-1) \times n}(R)$. Then $R_{\Sigma}$ is a division ring which coincides with the universal field of fractions of $R$ by [7, Corollary 4.5.9], which is, by definition, the free field [ $\mathbf{7}$, p. 224]. Linnell proved in [23] that the division closure of $R$ in $\mathcal{U}(H)$ is precisely $R_{\Sigma}$.

The reduced $C^{*}$-algebra $C_{r}^{*}(G)$ of a group $G$ is the norm-closure of $\mathbb{C}[G]$ in $B\left(\ell^{2}(G)\right)$. We have an inclusion $C_{r}^{*}(G) \subseteq \mathcal{N}(G)$. Let us denote by $S(G)$ the division closure of $\mathbb{C}[G]$ in $\mathcal{N}(G)$, which coincides with the division closure of $\mathbb{C}[G]$ in $C_{r}^{*}(G)$.

For a free group $H$, Duchamp and Reutenauer computed $S(H)$ in $[\mathbf{9}]$, answering a question of Alain Connes. Let $\Gamma=(V, E)$ be the Cayley graph of a finitely generated free group $H$ with respect to a free set of generators $X$. Recall that $V=H$ and $E=H \times X$. There exists a bijection $\pi: H \rightarrow E \cup\{*\}$ such that, for $g \in H$,

$$
\{h \in H \mid \pi(g h) \neq g \pi(h)\}
$$

is finite. This bijection sends each non-identity element $g \in H$ to the first edge in the path from $g$ to 1 , and sends 1 to $*$. Therefore we get a unitary $P: \ell^{2}(H) \rightarrow \ell^{2}(E) \oplus \mathbb{C}$, and one can check easily that the commutator $[P, a]$ is a finite rank operator for all $a \in \mathbb{C}[H]$.

For a subalgebra $B \subseteq B\left(\ell^{2}(H)\right)$, we define

$$
B_{\text {fin }}=\{a \in B \mid[P, a] \text { has finite rank }\} .
$$

In $\left[8\right.$, p. 342, Remarque 3], Connes conjectured that $C_{r}^{*}(H)_{\text {fin }}$ coincides with the rational closure of $\mathbb{C}[H]$ in $C_{r}^{*}(H)$. Duchamp and Reutenauer proved in $[\mathbf{9}]$ the stronger statement that $S(H)=$ $\mathcal{N}(H)_{\text {fin. }}$. A similar result was then obtained by Linnell in [24] for the division closure $D(H)$ of $\mathbb{C}[H]$ in $\mathcal{U}(H)$. In particular, Linnell proved that $S(H)=D(H) \cap \mathcal{N}(H)$ and that $D(H)$ is the classical ring of quotients of $S(H)$. This equality does not hold in general, in fact if $G$ is a free abelian group of rank 2, then $D(G) \cap \mathcal{N}(G)$ is not contained in $C_{r}^{*}(G)=C\left(\mathbb{T}^{2}\right)$, where $\mathbb{T}^{2}$ is the 2-torus. This follows from the computations in $[\mathbf{2 0}$, p. 362].

Taking the $C^{*}$-completion of $D(G)_{b}=D(G) \cap \mathcal{N}(G)$ one obtains a sort of $C^{*}$-algebra of quotients of $C_{r}^{*}(G)$, determined by its dense *subalgebra $\mathbb{C}[G]$. It would be interesting to compute this $C^{*}$-algebra 
in specific situations. In particular, it seems plausible that it does not contain non-trivial idempotents in the case where $G$ is a torsionfree group in the Linnell class. This is the case when $G$ is the free abelian group of rank 2 , since $\overline{D(G)_{b}}$ is contained in the $C^{*}$-algebra $\lim _{U \in \mathcal{F}} C(U)$, where $\mathcal{F}$ is the family of sets of the form $\mathbb{T}^{2} \backslash F$, for finite $F$.

\section{REFERENCES}

[1] P. Ara and M. Mathieu, A local version of the Dauns-Hofmann theorem, Math. Z. 208 (1991), 349-353.

[2] P. Ara and M. Mathieu, A simple local multiplier algebra, Math. Proc. Cambridge Phil. Soc. 126 (1999), 555-564.

[3] P. Ara and M. Mathieu, Local multipliers of $C^{*}$-algebras, Monographs in Mathematics, Springer-Verlag, London 2003.

[4] K. I. Beidar, W. S. Martindale and A. V. Mikhalev, Rings with generalized identities, Marcel Dekker, New York, 1996.

[5] S. K. Berberian, Baer *-rings, Grundlehren Math. Wiss., Vol. 195, SpringerVerlag, Berlin Heidelberg, New York, 1972.

[6] S. K. Berberian, The maximal ring of quotients of a finite von Neumann algebra, Rocky Mountain J. Math. 12 (1982), 149-164.

[7] P. M. Cohn, Skew Fields, Theory of General Division Rings, Encyclopedia of Mathematics and its applications, Vol. 57, Cambridge University Press, Cambridge, 1995.

[8] A. Connes, Noncommutative geometry, Academic Press, New York, 1994.

[9] G. Duchamp and C. Reutenauer, Un critère de rationalité provenant de la géométrie non commutative, Invent. Math. 128 (1997), 613-622.

[10] G. A. Elliott, Derivations determined by multipliers on ideals of a $C^{*}$-algebra, Publ. Res. Inst. Math. Sci. 10 (1975), 721-728.

[11] G. A. Elliott, Automorphisms determined by multipliers on ideals of a $C^{*}$ algebra, J. Funct. Anal. 23 (1976), 1-10.

[12] N. J. Fine, L. Gillman and J. Lambek, Rings of quotients of rings of functions, McGill Univ. Press, Montreal, 1966.

[13] M. Frank, Injective envelopes and local multipliers of $C^{*}$-algebras, Int. Math. J. 1 (2002), 611-620.

[14] M. Frank and V. I. Paulsen, Injective envelopes of $C^{*}$-algebras as operator modules, Pacific J. Math., to appear.

[15] L. Gillman and M. Jerison, Rings of continuous functions, Springer-Verlag, Berlin, 1976. 
[16] K. R. Goodearl, Notes on real and complex $C^{*}$-algebras, Shiva Publ. Ltd., Nantwich, 1982.

[17] M. Hamana, Injective envelopes of $C^{*}$-algebras, J. Math. Soc. Japan 31 (1979), 181-197.

[18] M. Hamana, Regular embeddings of $C^{*}$-algebras in monotone complete $C^{*}$ algebras, J. Math. Soc. Japan 31 (1979), 181-197.

[19] M. Hamana, The centre of the regular monotone completion of a $C^{*}$-algebra, J. London Math. Soc. 26 (1982), 522-530.

[20] D. Handelman, Rings with involutions as partially ordered groups, Rocky Mountain J. Math. 11 (1981), 337-381.

[21] T. Y. Lam, Lectures on Modules and Rings, GTM 189, Springer-Verlag, 1999.

[22] S. Lanning, The maximal symmetric ring of quotients, J. Algebra 179 (1996), $47-91$.

[23] P. Linnell, Division rings and group von Neumann algebras, Forum Math. 5 (1993), 561-576.

[24] P. Linnell, A rationality criterion for unbounded operators, J. Funct. Anal. 171 (2000), 115-123.

[25] M. Mathieu, On $C^{*}$-algebras of quotients, Semesterbericht Funktionalanalysis 16 (1989), 107-120.

[26] M. Mathieu, How to solve an operator equation, Publ. Mat. 36 (1992), 743-760.

[27] M. Mathieu, The cb-norm of a derivation, in Algebraic methods in operator theory (R. E. Curto and P. E. T. Jørgensen, eds.), Birkhäuser, Basel Boston, 1994, pp. 144-152.

[28] M. Mathieu, Representation theorems for some classes of operators on $C^{*}$ algebras, Irish Math. Soc. Bull. 41 (1998), 44-56.

[29] M. Mathieu, Ten years of local multipliers, Irish Math. Soc Bull. 43 (1999), 64-69.

[30] G. J. Murphy, $C^{*}$-algebras and operator theory, Academic Press, Boston, 1990.

[31] F. J. Murray and J. von Neumann, On rings of operators, Ann. of Math. 37 (1936), 116-229.

[32] D. S. Passman, Computing the symmetric ring of quotients, J. Algebra 105 (1987), 207-235.

[33] D. S. Passman, Infinite crossed products, Academic Press, Boston, 1989.

[34] G. K. Pedersen, Approximating derivations on ideals of $C^{*}$-algebras, Invent. Math. 45 (1978), 299-305.

[35] G. K. Pedersen, $C^{*}$-algebras and their automorphism groups, Academic Press, London, 1979. 
[36] G. K. Pedersen, Analysis Now, Springer-Verlag, New York, 1989.

[37] D. W. B. Somerset, The local multiplier algebra of a $C^{*}$-algebra, II, J. Funct. Anal. 171 (2000), 308-330.

[38] B. Stenström, Rings of quotients, Grundl. d. math. Wiss. 217, Springer-Verlag, Berlin, 1975.

[39] I. Vidav, On some *-regular rings, Publ. Inst. Math. Acad. Serbe Sci. 13 (1959), 73-80.

Pere Ara,

Departament de Matemàtiques,

Universitat Autònoma de Barcelona,

E-08193 Bellaterra (Barcelona),

Spain

para@mat.uab.es

Received on 5 November 2001 and in revised form on 20 March 2003. 